\title{
Promoção da saúde de familiares cuidadores de praticantes de equoterapia: um relato de experiência
}

\author{
Health promotion of family caregivers of hippotherapy practitioners: an experience report \\ Promoción de la salud de los familiares cuidadores de practicantes de hipoterapia: relato \\ de experiencia
}

Marjana Pivoto Reginaldo ${ }^{1 *}$, Sandra Ost Rodrigues ${ }^{1}$, Claudete Moreschi ${ }^{1}$, Lenise Dutra da Silva ${ }^{1}$, Liane Bahú Machado².

\section{RESUMO}

Objetivo: Desenvolver ações de promoção da saúde com os familiares cuidadores de crianças com necessidades especiais que frequentam a Equoterapia. Relato de experiência: Acerca de uma Prática Assistencial realizada durante o mês de setembro e outubro de 2021 em um Centro de Equitação Cristal localizado de um município de pequeno porte da região centro-oeste do Rio Grande do Sul. Os encontros foram realizados semanalmente de acordo com a disponibilidade e aceitação por parte da equipe e dos participantes, participaram da prática 10 familiares cuidadores. Os encontros foram divididos em cinco momentos, sendo realizados nas segundas-feiras durante o turno da manhã e tarde com duração de aproximadamente 60 minutos. Considerações finais: Foi possível perceber a importância do enfermeiro na promoção da saúde, no acolhimento, na formação de vínculo e de uma assistência continuada com os familiares dos praticantes de Equoterapia em função das demandas que eles enfrentam no dia a dia. Podese perceber o quanto é necessário ter um olhar integral e atento as famílias dos praticantes de Equoterapia e comprovou-se unanimamente, o quanto os mesmos se sentiram acolhidos, cuidados e principalmente valorizados pelos profissionais durante esta prática assistencial.

Palavras-chave: Crianças com deficiência, Terapias complementares, Familiares cuidadores, Promoção da saúde.

\begin{abstract}
Objective: To develop health promotion actions with family caregivers of children with special needs who attend Riding Therapy. Experience report: About a Care Practice carried out during the months of September and October 2021 in a Crystal Riding Center located in a small town in the midwest region of Rio Grande do Sul. The meetings were held weekly according to with the availability and acceptance by the team and the participants, 10 family caregivers participated in the practice. The meetings were divided into five moments, being held on Mondays during the morning and afternoon shifts, lasting approximately 60 minutes. Final considerations: It was possible to perceive the importance of nurses in health promotion, reception, bonding and continued assistance with the family members of Riding Therapy practitioners due to the demands they face on a daily basis. It can be seen how much it is necessary to have a comprehensive and attentive look at the families of practitioners of Hippotherapy and it was unanimously proved how much they felt welcomed, cared for and especially valued by professionals during this care practice.
\end{abstract}

Keywords: Children with disabilities, Complementary therapies, Family caregivers, Health promotion.

1 Universidade Regional do Alto Uruguai e das Missões Campus Santiago (URI), Santiago - RS.

*E-mail: marjanapivotto@gmail.com

2 Universidade Federal de Santa Maria (UFSM), Santa Maria - RS. 


\section{RESUMEN}

Objetivo: Desarrollar acciones de promoción de la salud con cuidadores familiares de niños con necesidades especiales que asisten a Equitación Terapia. Informe de experiencia: Sobre una Práctica de Cuidado realizada durante el mes de septiembre y octubre de 2021 en un Crystal Riding Center ubicado en una pequeña ciudad en la región centro-oeste de Rio Grande do Sul. Las reuniones se realizaron semanalmente de acuerdo con la disponibilidad y aceptación por parte de del equipo y de los participantes, 10 cuidadores familiares participaron de la práctica. Las reuniones se dividieron en cinco momentos, realizándose los días lunes en los turnos de mañana y tarde, con una duración aproximada de 60 minutos. Consideraciones finales: Fue posible percibir la importancia del enfermero en la promoción de la salud, acogida, vínculo y asistencia continuada con los familiares de los practicantes de Equitación Terapéutica debido a las exigencias que enfrentan en el día a día. Se ve cuánto es necesaria una mirada integral y atenta a las familias de los practicantes de Hipoterapia y se comprobó unánimemente cuánto se sintieron acogidos, cuidados y especialmente valorados por los profesionales durante esta práctica asistencial.

Palabras clave: Niños con discapacidad, Terapias complementarias, Cuidadores familiares, Promoción de la salud.

\section{INTRODUÇÃO}

A utilização do cavalo como uma terapia é conhecida no Brasil por Equoterapia, essa tem se mostrado em estudos que pode aperfeiçoar o controle postural e o equilíbrio de crianças com necessidades especiais. Ao cavalo se deslocar o movimento do seu passo se desloca ao centro de gravidade em três planos: transverso, sagital e frontal, parecidos ao movimento pélvico do ser humano durante a marcha. Dessa maneira, desloca o centro de massa do praticante, favorecendo a propriocepção, com uma alta estimulação sensorial e promovendo dessa forma reações de alinhamento e equilíbrio postural entre outros benefícios (ARAUJO AE, et al., 2010).

A terapia com cavalos tem um grande campo de atuação com pacientes portadores de deficiência sensoriais, mentais e motoras. A execução dessa prática terapêutica é desenvolvida por uma equipe multidisciplinar na qual a equipe idealiza o plano de cuidado de acordo com a necessidade e capacidade de cada praticante (SILVA KVLG, et al., 2018). Segundo a Associação Nacional de Equoterapia (ANDE-BRASIL) (2021), "O Praticante de Equoterapia" é a terminologia empregada para caracterizar um indivíduo com deficiência e/ou com necessidades especiais quando o mesmo está em atividades equoterápicas. Nesta prática, o elemento do recurso participa de sua reabilitação, enquanto proporciona a interação com o seu cavalo.

Nesse sentido, os familiares cuidadores dessas crianças com necessidades especiais vivenciam uma rotina diária e sobrecarga física e psicológica, o que pode levar ao descuido com a própria saúde e afetar a sua qualidade vida, refletindo de forma negativa no seu bem-estar, do praticante e da sua família (PIMENTA RA, et al., 2010). Geralmente, o mesmo sente-se despreparado diante das limitações das necessidades da criança, que acaba influenciando no estabelecimento dessa relação. Sendo assim, percebe-se uma grande necessidade de preocupar-se não só com o praticante, mas também de cuidar daquele que dedica grande parte do seu tempo aos cuidados da criança (SOUZA V, et al., 2011).

Como parte da equipe multiprofissional que atua na Equoterapia, o enfermeiro tem um papel de extrema importância na assistência dos familiares cuidadores e dessa forma deve elaborar junto a eles um plano de cuidados baseado na promoção da saúde dos mesmos por meio de educação em saúde (LANDEIRO MJL, et al., 2016). A mediação dos cuidados de enfermagem nos diferentes cenários de prática pode contribuir para a eficácia das ações educativas, uma vez que os familiares se sentem capazes de realizar os cuidados das crianças no domicílio através das relações entre profissionais e a família, sendo assim podendo visar uma qualidade de vida melhor para ele mesmo no seu dia a dia (OLIVEIRA SG e RESSEL LB, 2010).

Esta prática assistencial justificou-se pela vivência no ambiente em que foram realizadas as práticas de Equoterapia, percebeu-se uma necessidade no cuidado aos familiares cuidadores, que na maioria das vezes 
mostraram-se carentes e cansados. Sendo assim, objetivou-se com essa prática assistencial desenvolver ações da promoção de saúde com os familiares cuidadores de crianças com necessidades especiais que frequentam a Equoterapia, no sentido de compreender que o enfermeiro é um profissional fundamental no contexto da promoção de saúde, acolhimento e criação de vínculo.

\section{RELATO DE EXPERIÊCIA}

Será relatada uma Prática Assistencial realizada durante os meses de setembro e outubro de 2021 em um Centro de Equitação de um município de pequeno porte da região centro-oeste do Rio Grande do Sul. Os encontros foram realizados semanalmente de acordo com a disponibilidade e aceitação por parte da equipe e dos participantes, participaram da prática 10 familiares cuidadores. Os encontros foram divididos em cinco momentos, sendo realizados nas segundas-feiras durante o turno da manhã e tarde com duração de aproximadamente 60 minutos.

Para dar início a atividade prática, foi realizada uma visita ao local, para que fosse possível conhecer a rotina. Posteriormente, foram realizados alguns encontros individuais com os 10 familiares no decorrer das sessões de equoterapia para dar início a criação de vínculos. Após, foi entregue um convite com um bombom para os mesmos que aceitaram participar dos encontros e práticas propostas. Logo, a prática assistencial realizada aconteceu da seguinte maneira:

No primeiro encontro com os familiares, foi separada e organizada uma sala para recepcionar os familiares individualmente, com todos os cuidados e protocolos que esse cenário pandêmico exigia. Foi elaborado um instrumento de consulta de enfermagem com algumas perguntas básicas referentes as suas rotinas. Foi verificado os sinais vitais, alguns estavam um pouco queixosos e cansados devido a sobrecarga e a responsabilidade que carregam no dia a dia, intensificando que no cuidado geralmente é somente direcionado a criança com necessidades especiais.

Conforme os relatos, foram sendo realizadas orientações de cuidados com a saúde, como: a importância do autocuidado e de uma rotina na qual eles também pudessem cuidar um pouco de si, a relevância da ingesta hídrica diária, de uma alimentação saudável e da prática de atividades físicas visando o bem-estar físico e mental, sobre o cuidado para o uso correto das medicações conforme orientado, realização de exames de rotina, buscar algum tipo de terapia, se necessário e fazer algo que de prazer e que gostem, priorizando um tempo para lazer. Era visível a interação, o quanto estavam sentindo-se à vontade para relatar sobre suas rotinas e também atentos as orientações que foram sendo feitas, também trouxeram as dificuldades que alguns encontravam para conseguir ter um pouco de tempo pra si e mostravam-se felizes e satisfeitos com a consulta. Ao final de cada consulta, os participantes demonstravam satisfação e interesse em dar continuidade nos encontros.

No segundo encontro, participaram nove familiares de forma individual, foi realizada uma oficina de plantas medicinais, afim de que os mesmos adquirissem conhecimento sobre as plantas e também relatassem sobre quais faziam uso ou tinham conhecimento. Foram apresentados diversos tipos de plantas e as maneiras de utilizá-las, através de uma apresentação em formato de PowerPoint. Foi constatado, que a maioria dos familiares faziam o uso incorreto de algumas plantas medicinais, por exemplo: o chá de capim-santo era preparado por decocção, o que como consequência, acaba perdendo o seu efeito terapêutico. Houve relatos de que alguns estavam tentando utilizar os chás para deixar de fazer uso certas medicações antidepressivas, oportunizando um momento de troca de conhecimentos e experiências.

Para o terceiro encontro, ofertou-se a reflexoterapia palmar e a musicoterapia afim de "acalmar a mente e relaxar o corpo". Foi abordado sobre os benefícios das terapias propostas e apresentado uma imagem ilustrativa dos pontos reflexos localizados nas mãos. Participaram oito familiares de forma individual, após o pedido para que eles ficassem numa posição confortável, foi colocado a música e iniciado a prática da reflexoterapia palmar utilizando um óleo hidratante de erva-doce. A partir disso, a cada término da terapia os familiares relataram o quanto foi prazeroso e como o tempo tinha passado rápido, que o momento havia proporcionado uma sensação de paz, descanso físico e mental, uma desconexão do mundo exterior, da rotina, das preocupações e dos afazeres diários. 
No quarto encontro o tema explanado foi a arteterapia e a musicoterapia, seguindo as sugestões dos participantes. Neste dia estavam sete familiares presentes. Foi apresentado vários modelos de mandalas para colorir, disponibilizado diferentes tipos de lápis de cor. A participação dos familiares foi ativa no sentido de demonstrarem interesse pela temática. Também foi possível escutar alguns relatos a respeito do quanto eles gostavam de pintar e que fazia muito tempo que não tinham um momento assim, para se concentrar em algumas coisas que gostam de fazer, algumas vezes pintam com os filhos, mas que não se comparava com o momento ofertado.

O quinto encontro se sucedeu conforme algumas observações feitas durante os encontros anteriores, visto a relevância de fazer-se uma meditação guiada chamada "gratidão". Desse modo foi convidado os familiares individualmente. Eles sentaram em uma poltrona em posição confortável e diante disso foi explicado sobre os benefícios da meditação, esclarecido algumas dúvidas, após foram feitas as orientações para realização da meditação. Ao termino da atividade proposta foi ofertado um lanche partilhado para encerramento das práticas, foi entregue um cartão com um bombom para cada familiar como uma lembrança do momento.

\section{DISCUSSÃO}

É de extrema importância que as instituições de saúde, educação e a comunidade desenvolvam aliança entre a família e os serviços, com objetivo comum para os melhores interesses das crianças com necessidades especiais, além disso, precisam considerar as necessidades, os pontos fortes e fracos dessas famílias. Essas atividades podem ter impactos extensos e profundos nos aspectos físico, social, financeiro e emocional das famílias, os serviços de saúde são desafiados a apoiar e a acompanhar as famílias no atendimento a essas crianças (AMANDA LS, et al., 2020).

Como parte da equipe multiprofissional que trabalham na Equoterapia, o enfermeiro tem o papel de fundamental na assistência dos familiares cuidadores e dessa forma elaborar junto a eles um plano de cuidados baseado na promoção da saúde dos mesmos por meio de educação em saúde (LANDEIRO MJL, et al., 2016). A mediação dos cuidados de enfermagem nos diferentes cenários de prática pode contribuir para a eficácia das ações educativas, uma vez que os familiares sentem-se capazes de realizar os cuidados das crianças no domicílio através das relações entre profissionais e a família (OLIVEIRA SG e RESSEL LB, 2010).

Desse modo, destaca-se a relevância das ações de promoção da saúde, as mesmas devem ser estabelecidas em conjunto por meio das ferramentas de Educação em Saúde que evidenciam a autonomia, o empoderamento, autoconfiança, autocuidado. Acredita-se que cabe então, tanto aos profissionais de saúde quanto aos educadores, a sensibilização para a promoção do conhecimento e das discussões e, diante do exposto, é percebível a precisão de intervenções educativas. Tem-se, como papel da Enfermagem, a educação em saúde, bem como sua promoção e prevenção e, junto à equipe, exercer ações e projetos educativos, com o intuito de dar autonomia a esses familiares cuidadores, faz com que esse seja o autor e protagonista do seu cuidado nos diversos cenários da saúde (SILVA KVLG, et al., 2018).

A mãe, que na maioria dos casos é a cuidadora principal, assume grande parte do processo de cuidados necessários para a melhor qualidade de vida da criança com necessidade especial de saúde e foi identificado que elas podem contar apenas com o apoio de alguns familiares. Dessa forma, apesar desse apoio recebido, nota-se uma sobrecarga na vida do cuidador principal, que provoca um dano físico e emocional considerável (BARBOSA TA, et al., 2016). Essas dificuldades mencionadas demandam muito apoio e acolhimento dos profissionais de saúde, os quais devem aproveitar o encontro com a família para ajudarem a esclarecer dúvidas, acolherem e estarem sempre dispostos a fazer uma boa escuta. Sendo assim, o cuidado com as famílias que têm a experiência de ter um filho com necessidades especiais é importante para o fortalecimento delas no enfrentamento das dificuldades (INACIO ALR e PEIXOTO APGL, 2017).

Deste modo, a sobrecarga dos cuidadores/familiares pode estar relacionada à falta/ausência de escuta e orientações para assumir os cuidados diários com a criança sem deixar o seu próprio autocuidado de lado. Isto procede do fato de os próprios profissionais da área da saúde não estarem adequadamente preparados 
para o cuidado holístico e nas necessidades centradas na família cuidadora, enfim, caracterizar-se como uma rede de apoio com habilidade de oferecer um amparo emocional aos familiares e cuidadores, principalmente para as mães, responsáveis primordiais do cuidado íntegro dessas crianças (SEVERO VRG, et al., 2019).

O Conselho Federal de Enfermagem (COFEN), no Parecer Informativo 004/95, em 1995, distinguiu na profissão de Enfermagem, que é baseada na visão do ser humano como um todo, o constante interesse em utilizar práticas naturais no decorrer do tratamento do cliente, e também as concepções do Código de Ética dos Profissionais de Enfermagem, que possibilita o uso dessas práticas. Contudo, apenas no ano de 1997, por meio da Resolução 197, que o COFEN reconheceu essas terapias alternativas como qualificação e especialidade do profissional de enfermagem (COFEN, 1997).

Dentro desse processo de integração, o enfermeiro está dentro da equipe de terapeutas que atuam na Equoterapia possibilitando o reconhecimento da enfermagem, no qual sua participação pode ter bastante importância em relação aos familiares cuidadores dos praticantes, certificando assim a continuação do tratamento em casa. Devido ao profissional de enfermagem ter a habilidade de atuar com proximidade ao usuário, de maneira integralizada, ele pode direcionar as orientações para os cuidadores em relação às necessidades exigidas referente a patologia tratada, objetivando a identificação das formas de cuidado, auxiliando positivamente na satisfação das necessidades humanas básicas (STROCHEIN JR e RODRIGUES FCP, 2016).

As Práticas Integrativas e Complementares (PICs) podem ser conceituadas como tratamentos que utilizam recursos terapêuticos, que são baseados em conhecimentos tradicionais, aplicados principalmente para prevenção, tratamento e reabilitação de pacientes acometidos por patologias diversas (MINISTÉRIO DA SAÚDE, 2006). Também é evidente a acolhida das PICs em serviços públicos de saúde, onde obteve a regulamentação do Ministério da Saúde, na perspectiva de estímulo à propagação dessas práticas (ANDRADE JT e COSTA LFA, 2010). Dentre as PICs incluídas na Política Nacional de Práticas Integrativas e Complementares, ofertadas pelo SUS (PNPIC-SUS), podemos citar algumas como acupuntura, fitoterapia, reiki, meditação, musicoterapia, reflexoterapia, arteterapia, yoga, entre outras práticas (MINISTÉRIO DA SAÚDE, 2006). É válido afirmar que o campo das PICs, no Brasil atual, pode ser descrito como um fenômeno ascendente, com grande mercado de trabalho e visibilidade (ANDRADE JT e COSTA LFA, 2010).

Foi possível perceber a importância do enfermeiro na promoção da saúde, no acolhimento, na formação de vínculo e de uma assistência continuada com os familiares dos praticantes de Equoterapia em função das demandas que eles enfrentam no dia a dia. Pode-se perceber o quanto é necessário ter um olhar integral e atento as famílias dos praticantes de Equoterapia e comprovou-se unanimamente, o quanto os mesmos se sentiram acolhidos, cuidados e principalmente valorizados pelos profissionais durante esta prática assistencial. Evidenciou-se a relevância desta temática para a promoção de saúde dos usuários, bem como sugere-se que a mesma seja cada vez mais aprofundada e reconhecida objetivando alcançar maiores serviços.

\section{REFERÊNCIAS}

1. AMANDA LS, et al. Atenção primária à saúde para atendimento às famílias de crianças com necessidades especiais. Biblioteca Virtual em Saúde, 2020.

2. ASSOCIAÇÃO NACIONAL DE EQUOTERAPIA (ANDE-BRASIL), 2021. Disponível em http://equoterapia.org.br/. Acesso em 08 de junho de 2021.

3. ANDRADE JT, COSTA LFA. Medicina complementar no SUS: Práticas Integrativas e Complementares sob a luz da Antropologia médica. Saúde Soc., São Paulo, 2010; 19(3): 497- 508.

4. ARAUJO AE, et al. A equoterapia no tratamento de crianças com paralisia cerebral no Nordeste do Brasil. Revista Fisioterapia Brasil, 2010; 11: 4-8.

5. BARBOSA TA, et al. Rede de apoio e apoio social às crianças com necessidades especiais de saúde. Revista Rene. $2016 ; 17(1): 60-6$.

6. CONSELHO FEDERAL DE ENFERMAGEM (COFEN). Resolução 197. Estabelece e reconhece as terapias alternativas como especialidade e/ou qualificação do profissional de Enfermagem. In: Conselho Regional de Enfermagem. Documentos básicos de enfermagem. São Paulo; 1997. Disponível em: http://www.cofen.gov.br/resoluocofen-1971997_4253.html. Acesso em 19 de novembro de 2021. 
7. INÁCIO ALR, PEIXOTO APGL. A assistência de enfermagem e o cuidado familiar às crianças com necessidades especiais de saúde: uma revisão integrativa. Rev. Aten. Saúde, 2017; 15(53): 87-94.

8. LANDEIRO MJL, et al. Percepção dos enfermeiros sobre Dificuldades e necessidades informacionais dos familiares cuidadores de pessoa dependente. Revista Texto Contexto Enfermagem, 2016; 25(1).

9. MINISTÉRIO DA SAÚDE. Departamento de Atenção Básica. Política Nacional de Práticas Integrativas e Complementares e Complementares no SUS - PNPIC-SUS. Brasília - DF: Brasil, 2006. Disponível em: http://dtr2004.saude.gov.br/dab/publicacoes.php. Acesso em 19 de novembro de 2021.

10. OLIVEIRA SG, RESSEL LB. Grupos de adolescentes na prática de enfermagem: um relato de experiência. Revista Ciência, Cuidado e Saúde, 2010; 9(1): 144-148.

11. PIMENTA RA, et al. Avaliação da qualidade de vida e sobrecarga de cuidadores de pessoas com deficiência intelectual. Revista. Brasileira de Ciências da Saúde, 2010; 14(3): 69-76.

12. SILVA KVLG, et al. Training of adolescente multipliers from the perspective of health promotion core competencies. Revista Brasileira de Enfermagem, 2018; 71(1): 98-105.

13. SEVERO VRG, et al. Conhecimento prévio de cuidadoras de crianças com necessidades especiais de saúde: uma abordagem freiriana. Ciência, Cuidado E Saúde, 2019; 18(3).

14. SOUZA V, et al. Psicologia e equoterapia: conhecendo as dificuldades enfrentadas pelos cuidadores de praticantes. Revista Cientifica da Faminas, 2011; (7)3.

15. STROCHEIN JR, RODRIGUES FCPA. Percepção dos familiares e da equipe sobre o atendimento as Crianças com Necessidades Especiais em um centro de Equoterapia. Revista Vivências. 2016; (12)23: 16-32. 